Correspondence

David J. Richardson

d.richardson@uea.ac.uk
Received 22 October 2007

Revised 11 January 2008

Accepted 14 January 2008

\section{A combination of cytochrome $c$ nitrite reductase (NrfA) and flavorubredoxin (NorV) protects Salmonella enterica serovar Typhimurium against killing by $\mathrm{NO}$ in anoxic environments}

\author{
Paul C. Mills, ${ }^{1}$ Gary Rowley, ${ }^{2}$ Stephen Spiro, ${ }^{3}$ Jay C. D. Hinton ${ }^{2}$ \\ and David J. Richardson ${ }^{1}$
${ }^{1}$ School of Biological Sciences, University of East Anglia, Norwich NR4 7TJ, UK
${ }^{2}$ Molecular Microbiology Group, Institute of Food Research, Norwich NR4 7UA, UK
${ }^{3}$ Department of Molecular and Cell Biology, University of Texas at Dallas, 800 West Campbell Road, Richardson, TX 75080, USA

\begin{abstract}
The enteric bacterium Salmonella enterica serovar Typhimurium is a pathogen that is highly adapted for both intracellular and extracellular survival in a range of oxic and anoxic environments. The cytotoxic radical nitric oxide (NO) is encountered in many of these environments. Protection against NO may involve reductive detoxification in low-oxygen environments, and three enzymes, flavorubredoxin (NorV), flavohaemoglobin ( $\mathrm{HmpA}$ ) and cytochrome $c$ nitrite reductase (NrfA), have been shown to reduce NO in vitro. In this work we determined the role of these three enzymes in NO detoxification by Salmonella by assessing the effects of all eight possible combinations of norV, $h m p A$ and $n r f A$ single, double and triple mutations. The mutant strains were cultured and exposed to NO following either glucose fermentation (when nitrite reductase activity is low), or anaerobic respiration (when nitrite reductase activity is high). Wild-type cultures were more sensitive to the addition of a pulse of NO when grown under fermentative conditions compared with anaerobic respiratory conditions. Analysis of the mutant strains suggested an important additive role for both NorV and NrfA in both environments, since the norV nrfA mutant could not grow after $\mathrm{NO}$ addition. The results also suggested a minor role for $\mathrm{HmpA}$ in anaerobic detoxification of NO under the two growth conditions, and a larger role for $\mathrm{HmpA}$ in aerobic $\mathrm{NO}$ detoxification was confirmed. Activity assays and measurements of NO consumption showed that increased nitrite reductase activity correlates with an elevated capacity for NO reduction by intact cells. Taken together, the results reveal a combined role for NorV and NrfA in NO detoxification under anaerobic conditions, and highlight the influence that growth conditions have on the sensitivity to NO of this pathogenic bacterium.
\end{abstract}

\section{INTRODUCTION}

The highly reactive free radical nitric oxide $(\mathrm{NO})$ is encountered by bacteria in diverse environments. NO can be produced endogenously as an intermediate during the reduction of nitrite to nitrous oxide in denitrification, or as a by-product of the respiratory reduction of nitrite to ammonia. NO is also encountered by pathogens, since the molecule is produced by macrophages as a component of

Abbreviations: GSNO, S-nitrosoglutathione; $1_{50}$, concentration causing a $50 \%$ decrease in cell viability; SNP, sodium nitroprusside.

Three supplementary figures showing the effects of NO on the aerobic and anaerobic growth curves, with glucose or glycerol as the carbon source, of the eight $S$. Typhimurium strains examined in this study are available with the online version of this paper. the host innate immune response (Weinberg, 1999). Many bacteria are unable to withstand the combined actions of $\mathrm{NO}$ and superoxide, but a few, including Salmonella, are able to survive and eventually replicate within the macrophage (Vazquez-Torres \& Fang, 2001). It has been shown that the combined actions of inducible nitric oxide synthase (iNOS) and phagocyte oxidase (Phox) are important in bacterial killing and clearance by macrophages (Shiloh et al., 1999). In addition, Salmonella possesses enzymes capable of metabolizing NO, both aerobically and anaerobically. Consequently, much research has been undertaken to understand the mechanisms of in vitro $\mathrm{NO}$ detoxification, but with some contrasting results.

The cytoplasmic flavohaemoglobin ( $\mathrm{HmpA})$ enzyme has a well characterized NO dioxygenase activity in both 
Escherichia coli (Gardner et al., 1998; Hausladen et al., 1998; Membrillo-Hernandez et al., 1999) and Salmonella (Crawford \& Goldberg, 1998). However, the physiological significance of the oxygen-independent $\mathrm{NO}$ reductase activity of HmpA is not fully understood. Purified HmpA is able to reduce $\mathrm{NO}$ to nitrous oxide under anoxic conditions (Kim et al., 1999), and a Salmonella hmpA mutant was sensitive to the NO-releaser $S$-nitrosoglutathione (GSNO) when cultured in an anaerobic environment (Crawford \& Goldberg, 1998). E. coli hmpA expression is induced anaerobically by different sources of NO, including acidified nitrite (Mukhopadhyay et al., 2004), GSNO (Flatley et al., 2005), an NO-saturated solution (Justino et al., 2005), and NO made as a byproduct of nitrite respiration (Bodenmiller \& Spiro, 2006). However, it has been argued that $\mathrm{HmpA}$ can only reduce NO anaerobically with a turnover number 100 fold less than that recorded for aerobic conditions (Mills et al., 2001). Furthermore, E. coli overexpressing hmpA have a high aerobic NO dioxygenase activity, but a very low NO reductase activity (Gardner \& Gardner, 2002). Recent results have demonstrated some contribution of HmpA to Salmonella virulence in mice (Bang et al., 2006), and to survival within macrophages (Bang et al., 2006; Gilberthorpe et al., 2007; Stevanin et al., 2002), suggesting that this enzyme is important for successful infection.

The E. coli flavorubredoxin (NorV) is an oxygen-sensitive NO reductase, which contributes to NO detoxification under anaerobic conditions (Gomes et al. 2002; Gardner \& Gardner, 2002; Gardner et al., 2002). The anaerobic growth of an E. coli nor $V$ mutant is impaired in the presence of $\mathrm{NO}$ under anaerobic conditions that require the activities of NO-sensitive enzymes (Gardner et al., 2002) and is sensitive to the $\mathrm{NO}^{+}$donor sodium nitroprusside (SNP) (Hutchings et al., 2002). The expression of norV is upregulated upon the addition of a variety of NO sources (Flatley et al., 2005; Justino et al., 2005; Mukhopadhyay et al., 2004; Pullan et al., 2007), and this is dependent on its regulator, NorR (Mukhopadhyay et al., 2004; Hutchings et al., 2002). Furthermore, norV is upregulated in macrophage-internalized Salmonella at a time that corresponds to the NO burst (Eriksson et al., 2003), but the loss of NorV does not reduce the ability of $E$. coli to survive within macrophages (Pullan et al., 2007).

Cytochrome $c$ nitrite reductase (NrfA), which catalyses the six-electron reduction of nitrite, is also thought to catalyse the anaerobic five-electron reduction of NO. Purified NrfA from E. coli (Costa et al., 1990) and Sulfurospirillum deleyianum (Stach et al., 2000) can reduce NO to ammonium, with rates comparable to those of $\mathrm{NO}$ reductases that belong to the family of bacterial respiratory NO reductases (Field et al., 2008). Interestingly, an E. coli $n r f A$ mutant showed an increased anaerobic sensitivity towards NO (Poock et al., 2002). The nrf operon is regulated by the NO-sensitive repressor NsrR in E. coli (Filenko et al., 2007), which provides a possible mechanism for the upregulation of $n r f$ expression by NO. However, other studies have found little increase in $n r f$ expression upon the addition of NO (da Costa et al., 2003; Pullan et al., 2007), suggesting that it is not highly NO-responsive, at least under the conditions used in those assays.

Despite many reports on the proteins involved in NO detoxification, there are still inconsistencies. Previous research on single norV and hmpA mutants has shown differing levels of bacterial growth inhibition by an anaerobic NO source (Gilberthorpe et al., 2007; Hutchings et al., 2002; Justino et al., 2005). The cause of these discrepancies could be twofold: first, the type of growth medium used; and second, the source of NO used. As well as releasing NO, some chemical sources also release other toxic compounds, such as cyanide (released by SNP), though they have the advantage of releasing NO over a prolonged period. The use of NO gas is desirable, since no other compounds are released, but has the disadvantage that the NO can potentially be degraded quickly in the presence of oxygen.

In this study we aimed to discover the contribution that NrfA and NorV make to the detoxification of NO by Salmonella under anaerobic growth conditions. We chose two types of growth medium, one of which promoted fermentative growth and has been reported to induce low levels of NrfA activity in E. coli [minimal glucose medium (MGM)], and the other of which promoted nitraterespiratory growth and induced high levels of NrfA activity in E. coli [minimal glycerol nitrate fumarate (GNF) medium] (Darwin et al. 1993). We found that HmpA, NorV and NrfA can all contribute to NO detoxification in particular environments, but that the anaerobic detoxification of $\mathrm{NO}$ required the combination of the NorV and NrfA enzymes. We suggest that Salmonella has evolved multiple NO-detoxification mechanisms to resist killing by $\mathrm{NO}$ under a wide range of environmental conditions.

\section{METHODS}

Bacterial strains, media and growth conditions. All studies were performed using wild-type Salmonella enterica serovar Typhimurium (S. Typhimurium) SL1344, and mutants (Table 1) were constructed using the method of Datsenko \& Wanner (2000). For general culture, bacteria were grown aerobically at $37{ }^{\circ} \mathrm{C}$ with shaking at 200 r.p.m. in $50 \mathrm{ml} \mathrm{LB}$ medium in a $250 \mathrm{ml}$ conical flask with $20 \mu \mathrm{g}$ chloramphenicol $\mathrm{ml}^{-1}$ (final concentration) and/or $50 \mu \mathrm{g}$ kanamycin $\mathrm{ml}^{-1}$ (final concentration) added where appropriate. For experimental analysis, bacteria were grown in a minimal salts medium (Pope \& Cole, 1984) supplemented with either $55 \mathrm{mM}$ glucose and $0.01 \%$ (w/v) casamino acids (MGM) or with $40 \mathrm{mM}$ glycerol, $22 \mathrm{mM}$ sodium nitrate, $40 \mathrm{mM}$ sodium fumarate and $0.01 \%(\mathrm{w} / \mathrm{v})$ casamino acids (GNF).

Preparation of NO solution. NO gas (Sigma-Aldrich), which had been scrubbed with $0.1 \mathrm{M} \mathrm{NaOH}$, was bubbled through an anaerobic preparation of water at $\mathrm{pH} 3$ until saturated. The saturated $(2 \mathrm{mM})$ solution was maintained under anaerobic conditions, mixed thoroughly and used immediately. 
Table 1. Strains used in this study

\begin{tabular}{|lll|}
\hline Strain & \multicolumn{1}{c|}{ Genotype } & \multicolumn{1}{c|}{ Source } \\
\hline S. Typhimurium SL1344 & Wild-type & Hoiseth \& Stocker (1981) \\
SL1344 norV & norV:: cat & This study \\
SL1344 nrfA & nrfA::kan & This study \\
SL1344 hmpA & nmpA::kan & This study \\
SL1344 norV hmpA & norf: :cat, hmpA:: kan $:$ kan, $\Delta h m p A$ & This study \\
SL1344 nrfA hmpA & norV::cat, nrfA:: kan & This study \\
SL1344 norV nrfA & norV::cat, nrfA:: kan, $\Delta h m p A$ & This study \\
SL1344 norV nrfA hmpA & & This study \\
\end{tabular}

Anaerobic growth. Either $1 \mathrm{ml}$ fresh MGM or $1 \mathrm{ml}$ GNF medium, in a $1 \mathrm{ml}$ cuvette, was inoculated with $5 \%(\mathrm{v} / \mathrm{v})$ of an overnight anaerobic culture grown in the same medium. Once inoculated, the cuvette was sealed and flushed with oxygen-free nitrogen to rid the system of oxygen. The cuvettes were incubated at $37{ }^{\circ} \mathrm{C}$ with periodic mixing to prevent settling. Growth was monitored by measuring the $\mathrm{OD}_{600}$. Once the cultures had reached an $\mathrm{OD}_{600}$ of $0.1, \mathrm{NO}$ was added to a final concentration of $40 \mu \mathrm{M}$ and growth was continued at $37{ }^{\circ} \mathrm{C}$.

Aerobic growth. A flask containing $50 \mathrm{ml}$ fresh MGM was inoculated with $1 \%(\mathrm{v} / \mathrm{v})$ of an overnight culture grown in the same medium. Cultures were incubated at $37{ }^{\circ} \mathrm{C}$ with shaking and growth was monitored by measuring the $\mathrm{OD}_{600}$. Once the culture reached an $\mathrm{OD}_{600}$ of 0.1 , NO was added to a final concentration of $200 \mu \mathrm{M}$ and monitoring of growth was continued.

Viability counts. Duplicate cultures of wild-type $S$. Typhimurium SL1344 and each mutant were grown anaerobically in MGM at $37{ }^{\circ} \mathrm{C}$. Once the $\mathrm{OD}_{600}$ reached $0.1,40 \mu \mathrm{M} N \mathrm{NO}$ was added to one culture and growth was continued. At $4 \mathrm{~h}$ post NO addition, samples were taken from each culture, which were serially diluted and plated onto LB agar containing the appropriate antibiotic(s). Following overnight growth at $37{ }^{\circ} \mathrm{C}$, the number of c.f.u. was determined. The percentage survival was calculated using cultures that had not been challenged with $\mathrm{NO}$ as the control.

Nitrite reductase assay. Cultures were harvested and the periplasmic fraction was isolated as described previously (Poock et al., 2002). Periplasmic fractions $(0.5 \mathrm{ml})$ in $10 \mathrm{mM}$ phosphate buffer $(\mathrm{pH} 7.5)$ and $1 \mathrm{mM}$ (final concentration) methyl viologen were added to a $1 \mathrm{ml}$ cuvette. The cuvette was sealed and the headspace degassed with oxygen-free nitrogen for $10 \mathrm{~min}$. Methyl viologen was reduced by the addition of a few microlitres of degassed $1 \mathrm{mM}$ sodium dithionite. Once the $A_{600}$ was stable between 1.5 and 2 units, potassium nitrite was added to a final concentration of $10 \mathrm{mM}$ to begin the reaction. This was followed by a decrease in $A_{600}$ and the specific activity was calculated from this rate, using a molar absorption coefficient of $13 \mathrm{mM}^{-1} \mathrm{~cm}^{-1}$ for reduced methyl viologen.

NO consumption assays. Following growth in MGM, $50 \mathrm{ml}$ bacterial culture was harvested by centrifugation at $10000 \mathrm{~g}$ for $25 \mathrm{~min}$. The resulting pellet was washed twice in fresh MGM and the final pellet was resuspended in $250 \mu \mathrm{l}$ fresh MGM to give a final density of approximately $1 \mathrm{~g} \mathrm{ml}^{-1}$. A $1.8 \mathrm{ml}$ volume of fresh MGM was added to the chamber of a Clark-type oxygen electrode (Hansatech Instruments). Once the signal was stable, $100 \mu \mathrm{l}$ of bacterial suspension was added. Following the removal of oxygen by bacterial respiration, $200 \mathrm{nmol} \mathrm{NO}$ was added, and the rate of NO consumption was followed on the trace.

\section{RESULTS}

\section{Sensitivity of $S$. Typhimurium wild-type and the hmpA, norV and nrfA mutants to NO during anaerobic growth in MGM}

Initial experiments were conducted in MGM, which was chosen because cells grown in this medium have a low level of endogenous nitrite reductase activity $(4 \pm 2 \mathrm{nmol}$ $\left.\mathrm{min}^{-1}\right)$. S. Typhimurium grows anaerobically in the absence of NO in MGM with a doubling time of $\sim 60 \mathrm{~min}$. In cultures to which $\mathrm{NO}$ was added (to a final concentration of $40 \mu \mathrm{M}$ ) a short $\sim 50 \mathrm{~min}$ period of growth inhibition was observed (see Table 2, Fig. 1 for four examples of growth curves, and Supplementary Fig. S1 for all eight growth curves). Under these conditions, single hmpA and nrfA mutants did not show an increased sensitivity to NO compared with the wild-type (Table 2). However, the norV mutant showed a longer period of growth inhibition ( $100 \mathrm{~min}$; Table 2 , Fig. 1$)$. These results suggest a key role for NorV-dependent NO detoxification under these anaerobic growth conditions, but do not reveal a clear role for either NrfA or HmpA. However, the norV strain does recover from $\mathrm{NO}$ addition, suggesting that another enzyme is capable of detoxifying the NO, possibly NrfA, HmpA or both.

Double mutants were constructed to establish the roles of the three NO-detoxification systems. Anaerobic growth of the $n r f A$ hmpA double mutant in MGM medium did not show increased sensitivity to NO compared with the wildtype (Table 2), but the norV hmpA mutant had a substantially increased growth inhibition period of $\sim 160$ min (Table 2), revealing the importance of NorV and HmpA for NO detoxification under these growth conditions. Most significantly, the norV nrfA double mutant showed a severe growth impediment upon NO addition, and did not recover (Table 2, Fig. 1; note that growth curves were monitored for a further $24 \mathrm{~h}$ beyond the time shown in Fig. 1 and recovery was still not observed). These data suggest that NorV and NrfA are the most important enzymes for anaerobic detoxification of NO during growth in MGM. In the absence of NorV and NrfA, HmpA is unable to protect Salmonella under the conditions tested, and apparently no other system can 
Table 2. Growth parameters for each mutant grown anaerobically in MGM

MGM is a minimal medium with glucose as the carbon source. The doubling times $\left(t_{\mathrm{d}}\right)$ pre and post NO addition are shown, as well as the period of growth inhibition, which was measured as the time between NO addition and a line extrapolated from the growth curve, following recovery, through the intercept of the $\mathrm{OD}_{600}$ at which NO was added. All data are the mean of at least three experiments and the SE is shown. Significant growth inhibition is indicated in bold type.

\begin{tabular}{|llcc|}
\hline Strain & $\boldsymbol{t}_{\mathbf{d}}(\mathbf{m i n})$ & $\boldsymbol{t}_{\mathbf{d}}$ post NO (min) & Growth inhibition period (min) \\
\hline S. Typhimurium SL1344 & $63.8 \pm 6.8$ & $62.4 \pm 10.6$ & $51.6 \pm 12.5$ \\
norV & $55.0 \pm 14.7$ & $72.3 \pm 5.0$ & $\mathbf{1 0 1 . 3} \pm \mathbf{2 7 . 3}$ \\
nrfA & $64.3 \pm 7.4$ & $64.0 \pm 6.6$ & $38.7 \pm 3.8$ \\
hmpA & $70.7 \pm 10.2$ & $79.7 \pm 5.5$ & $77.7 \pm 7.6$ \\
norV, $h m p A$ & $88.3 \pm 6.5$ & $94.7 \pm 7.8$ & $\mathbf{1 6 5 . 7} \pm \mathbf{3 6}$ \\
nrfA, $h m p A$ & $74.5 \pm 3.5$ & $88.5 \pm 5.5$ & $78.5 \pm 34.5$ \\
norV, $n r f A$ & $79.5 \pm 6.0$ & Not recordable & No recovery \\
norV, $n r f A, h m p A$ & $72.5 \pm 2.5$ & Not recordable & No recovery \\
\end{tabular}

facilitate growth recovery. This was confirmed by the essentially identical phenotypes following challenge with NO that were shown by the norV nrfA hmpA triple mutant and the nor $V$ nrfA double mutant (Table 2).

To assess the effect of $\mathrm{NO}$ on cell viability and to distinguish between bacteriostasis and toxicity, growth experiments were conducted in exactly the same manner as those described above, but NO was added to cultures at a range of different concentrations. Cultures were sampled after $240 \mathrm{~min}$ and viable counts determined. The results showed a concentration of NO that caused a $50 \%$ decrease in cell viability $\left(\mathrm{I}_{50}\right)$ of between $\sim 40$ and $\sim 60 \mu \mathrm{M}$ for wildtype cultures and the norV, nrfA, hmpA, norV hmpA and $n r f A$ hmpA mutants, but this is decreased to $\sim 5 \mu \mathrm{M}$ for the norV nrfA mutant and the triple mutant (Table 3). This hypersensitivity to $\mathrm{NO}$ underlies the inability of the nor $V$ $n r f A$ and triple mutant cultures to recover from the $40 \mu \mathrm{M}$ NO pulses administered in the experiments depicted in Fig. 1 and Table 2, since cell killing occurred at this level of
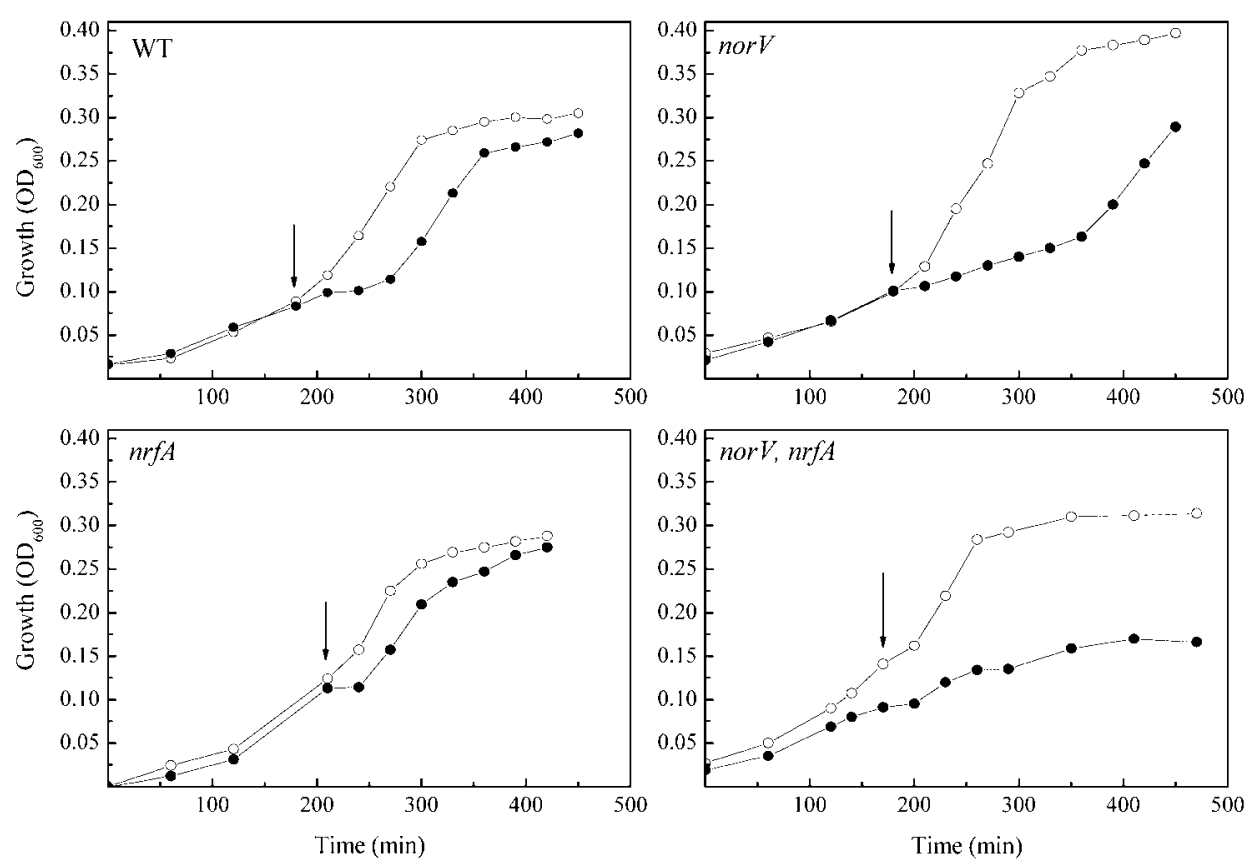

Fig. 1. Representative anaerobic growth curves of wild-type (WT) $S$. Typhimurium and three mutants in MGM, a minimal medium with glucose as the carbon source. Duplicate cultures were grown in the absence $(\bigcirc)$ and presence $(\bullet)$ of $40 \mu \mathrm{M} N O$. $\mathrm{NO}$ was added at the time indicated by the arrow and the data are representative of three independent experiments. Representative growth curves for all seven mutants are shown in Supplementary Fig. S1. 
Table 3. $I_{50}$ data for wild-type $S$. Typhimurium and NOdetoxification mutants grown anaerobically in MGM

MGM is a minimal medium with glucose as the carbon source. The $\mathrm{I}_{50}$ was calculated from the data presented in Fig. 2.

\begin{tabular}{|lc|}
\hline Strain & $\mathbf{I}_{\mathbf{5 0}}(\boldsymbol{\mu} \mathbf{M})$ \\
\hline S. Typhimurium SL1344 & 61 \\
norV & 54 \\
nrf & 53 \\
hmpA & 49 \\
norV, hmpA & 51 \\
nrfA, hmpA & 39 \\
norV, nrfA & 7 \\
norV, nrfA, hmpA & 4 \\
\hline
\end{tabular}

NO (Fig. 2). By contrast, the wild-type and the nor $V$, nrfA, hmpA, norV hmpA and nrfA hmpA mutants retained 50$80 \%$ viability at this NO concentration (Fig. 2), and the cultures were able to recover after a transient stasis (Table 2).

Taking these results together, we have discovered an important combined role for NorV and NrfA in NO detoxification during anaerobic growth of $S$. Typhimurium on MGM. These results are consistent with experiments conducted in E. coli (Gardner \& Gardner, 2002; Hutchings et al., 2002) that suggest that NorV is important in anaerobic NO detoxification, and that HmpA is not needed for protection against anaerobic NO attack. They also support the results of Poock et al. (2002), in suggesting that NrfA plays a role in anaerobic NO detoxification in E. coli. However, the results disagree with other reports which argue that HmpA is important for anaerobic NO detoxification in E. coli (Justino et al., 2005) and $S$. Typhimurium (Crawford \& Goldberg, 1998; Gilberthorpe et al., 2007). This variation may reflect differences in growth conditions and/or the source of NO. Some of these studies relied upon GSNO or SNP as the NO source, which were not used here due to the release of other toxic compounds.

\section{Correlation of cellular NO reduction capacity with nitrite reductase activity}

To gain further insight into the contribution of NorV, NrfA and HmpA to NO consumption during anaerobic growth on MGM, cells were removed from the cultures at various times after $\mathrm{NO}$ addition and the specific anaerobic $\mathrm{NO}$ consumption activity was measured. Relatively low NO consumption rates were measured before $\mathrm{NO}$ addition (Fig. 3a). This cellular activity increased over the next 180 min: after the cultures had recovered from the transient NO-induced, decreased growth rate, the specific NO reduction capacity was approximately eightfold greater than that prior to $\mathrm{NO}$ addition. This increase presumably reflects the NO-inducible synthesis or activation of enzymic systems capable of anaerobic NO reduction. The final specific NO reduction activity of the $n r f A$ strain was only $20 \%$ of that of the wild-type, indicating a significant contribution of NrfA activity to the overall rate of $\mathrm{NO}$ consumption.

The specific activity of nitrite reductase was low in periplasmic fractions of wild-type cells prior to $\mathrm{NO}$ addition, but increased following addition in a manner that mirrored the increase in NO consumption (Fig. 3b). This increase was not detected in the nrfA mutant, confirming that NrfA was responsible for most of the measurable nitrite reductase activity. Residual activity in the nrfA mutant could be due to non-specific nitrite reactions of other enzymes. These results suggest that NrfA

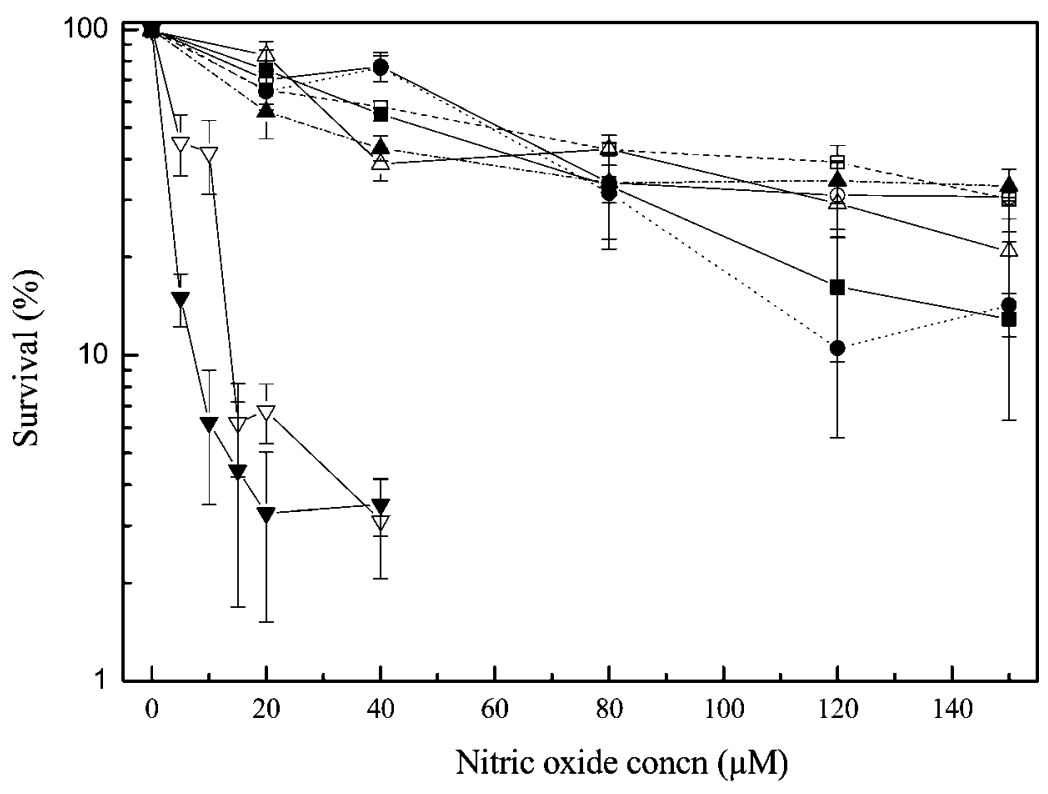

Fig. 2. Viability of wild-type $S$. Typhimurium SL1344 and mutant strains following NO addition to anaerobic liquid cultures. Viabilities were determined 240 min post NO addition, and are shown as a percentage of the values for control cultures from which NO was omitted. Results are the mean of three independent experiments and error bars indicate SE. $\bigcirc$, Wild-type strain; $\bullet$, norV strain; $\square$, nrfA strain; $\mathbf{\square}, h m p A$ strain; $\triangle, \operatorname{norV} h m p A$ strain; $\boldsymbol{\Delta}, \operatorname{nrf} A$ hmpA strain; $\nabla$, norV nrfA strain; $\boldsymbol{\nabla}$, norV nrfA hmpA strain. 

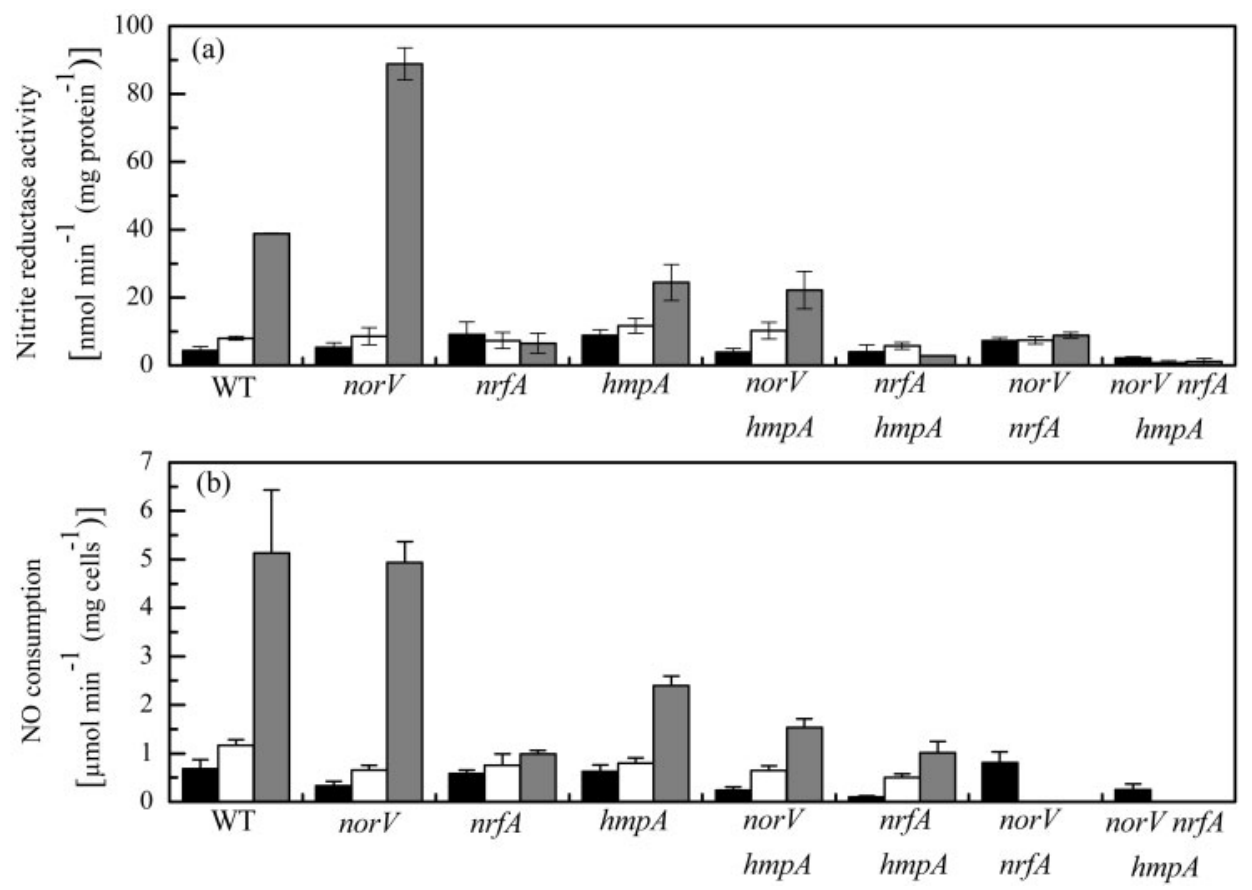

Fig. 3. Cellular NO consumption rates and nitrite reductase activity following addition of NO to cultures of $S$. Typhimurium grown anaerobically in MGM, a minimal medium with glucose as the carbon source. (a) Nitrite reductase activity of wild-type (WT) S. Typhimurium and mutants before and after NO addition. Samples were taken of each strain grown anaerobically in MGM before NO addition (black bars), 60 min after NO addition (white bars) and 180 min after NO addition (grey bars). NO was added to a final concentration of $40 \mu \mathrm{M}$ once the $\mathrm{OD}_{600}$ of the culture had reached 0.1 . Periplasmic fractions were prepared and assayed for nitrite reductase activity by the methyl viologen assay. Results are the average of three experiments and the SE is shown. (b) NO consumption rates of WT S. Typhimurium and mutants before and after NO addition. Samples were taken of each strain grown anaerobically in MGM before NO addition (black bars), 60 min after NO addition (white bars) and 180 min after NO addition (grey bars). NO was added to a final concentration of $40 \mu \mathrm{M}$ once the $\mathrm{OD}_{600}$ of the culture had reached 0.1 . Samples were washed and rates of NO consumption determined with a modified oxygen electrode. Results are the average of three experiments and the SE is shown. No rates could be measured for the norV nrfA double mutant and the norV $n r f A$ hmpA triple mutant after NO addition, because these cell cultures were killed by the NO addition, as determined by viable count assays.

accounts for most of the NO reduction capacity that is switched on following addition of $\mathrm{NO}$ to the bacterial cultures. Consistent with this view, a large increase in both nitrite reductase and $\mathrm{NO}$ reduction activity was still observed in the nor $V$ strain following $\mathrm{NO}$ addition. In the $h m p A$ strain the final nitrite reductase activity and the NO reduction activity were both around $50 \%$ of that of the wild-type (Fig. 3). The reason for this lower activity in the hmpA strain is not yet known, but the ratio of NO reduction activity to nitrite reductase activity remained similar to that of the wild-type. Accordingly, we suggest that most of the NO consumption activity of wild-type $S$. Typhimurium can be ascribed to NrfA. However, in the nrfA hmpA mutant a clear increase in $\mathrm{NO}$ reduction capacity did occur following $\mathrm{NO}$ addition, although the final level was only $20 \%$ of that of wild-type cells. This probably reflects NorV activity, and is similar to the residual capacity measured in the nrfA single mutant. Whilst no significant decrease in NO reduction capacity was measured in nor $V$ cells, the high background activity of
NrfA would make it difficult to measure a $20 \%$ contribution by NorV.

\section{Anaerobic growth of $S$. Typhimurium mutants and sensitivity to NO under NrfA-inducing conditions}

The experiments described above suggested that NrfA contributed to the anaerobic detoxification of NO by $S$. Typhimurium. To develop this finding, further experiments were conducted under conditions that have been reported to induce NrfA in E. coli, namely anaerobic growth in minimal medium with glycerol as a carbon source and nitrate and fumarate as respiratory electron acceptors (GNF medium; Darwin et al., 1993). The activity of $S$. Typhimurium nitrite reductase was assayed and compared following anaerobic growth in GNF or MGM medium. Nitrite reductase activity was increased by growth in GNF $\left.\left[170 \pm 15 \mathrm{nmol} \mathrm{min}{ }^{-1} \text { (mg protein }\right)^{-1}\right]$ by 40 -fold compared to MGM [4 $\pm 2 \mathrm{nmol} \mathrm{m^{-1 }}$ (mg protein) $\left.{ }^{-1}\right]$. 
Table 4. Growth parameters for each mutant grown anaerobically in GNF medium

GNF is a minimal medium with glycerol as the carbon source and nitrate and fumarate as electron acceptors. The doubling times $\left(t_{\mathrm{d}}\right)$ pre and post NO addition are shown, as well as the period of growth inhibition, which was measured as the time between NO addition and a line extrapolated from the growth curve, following recovery, through the intercept of the $\mathrm{OD}_{600}$ at which $\mathrm{NO}$ was added. All data are the mean of at least three experiments and the SE is shown. Significant growth inhibition is indicated in bold type.

\begin{tabular}{|lccc|}
\hline Strain & $\boldsymbol{t}_{\mathbf{d}}(\mathbf{m i n})$ & $\boldsymbol{t}_{\mathbf{d}}$ post NO (min) & Growth inhibition period (min) \\
\hline S. Typhimurium SL1344 & $87.4 \pm 17.7$ & $111.5 \pm 12.0$ & No inhibition \\
norV & $68.0 \pm 4.4$ & $116.7 \pm 17.5$ & No inhibition \\
$n r f A$ & $75.0 \pm 2.6$ & $103.7 \pm 10.7$ & $\mathbf{7 7 . 7} \pm \mathbf{1 3 . 1}$ \\
hmpA & $80.7 \pm 5.5$ & $104.7 \pm 6.1$ & No inhibition \\
norV, $h m p A$ & $113.7 \pm 7.1$ & $134.3 \pm 10.1$ & $\mathbf{1 2 3 . 7} \pm \mathbf{1 1 . 0}$ \\
nff, hmpA & $91.5 \pm 3.5$ & $126.0 \pm 24.0$ & No inhibition \\
norV, $n r f A$ & $110.4 \pm 9.2$ & Not recordable & No recovery \\
norV, $n r f A, h m p A$ & $81.7 \pm 9.9$ & Not recordable & No recovery \\
& & & \\
\hline
\end{tabular}

Similar results were observed for E. coli, with $n r f A$ expression being maximal during growth in GNF medium, and the addition of glucose causing repression of NrfA (Darwin et al., 1993).

In MGM-grown $S$. Typhimurium cells the anaerobic NO

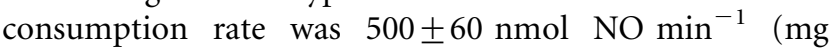
cells $)^{-1}$. Growth in GNF medium increased this rate by

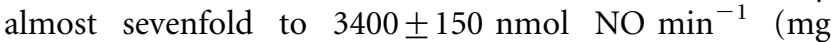
cells $)^{-1}$. Thus, there is a good correlation between the increase in nitrite reductase activity and the rate of NO consumption under GNF growth conditions.

To determine whether high levels of NrfA affected the ability to detoxify NO, the growth experiments conducted with MGM were repeated under NrfA-inducing conditions, in GNF medium. A key difference was observed: in contrast to the observations in the MGM growth experiments, wild-type Salmonella and the norV and hmpA mutants grown in GNF medium did not exhibit significant growth inhibition following addition of the NO (Table 4, Fig. 4; see Supplementary Fig. S2 for all eight growth curves). However, the nrfA mutant displayed a growth inhibition of $\sim 80 \mathrm{~min}$ (Table 4 ). These data show that in wild-type $S$. Typhimurium, a high nitrite reductase activity causes rapid NO detoxification and protects against growth inhibition following NO addition. However, NrfA is less able to protect in the absence of NorV or HmpA, since a double norV hmpA mutant showed a long period of growth inhibition ( $125 \mathrm{~min})$ that was absent in the single norV and hmpA mutants (Table 4). As with the MGM cultures, the norV nrfA double mutant stopped growing immediately upon $\mathrm{NO}$ addition and was unable to recover (Fig. 4), confirming that HmpA alone was unable to protect $S$. Typhimurium against anaerobic $\mathrm{NO}$ addition under the conditions tested here. These results demonstrate that NrfA can detoxify NO, and that prior synthesis of NrfA offers immediate protection to $S$. Typhimurium upon exposure to the cytotoxin.

\section{Aerobic growth of $S$. Typhimurium mutants and sensitivity to NO in MGM}

To determine the activity of the three enzymes under oxic conditions, aerobic growth was measured (Table 5, Fig. 5; see Supplementary Fig. S3 for all eight growth curves). A higher concentration of NO $(200 \mu \mathrm{M})$ was used in these experiments, since NO is labile in the presence of oxygen and has a short lifespan. To establish a function for each enzyme, the inhibition of wild-type cultures was used as a reference, and any role for HmpA, NorV or NrfA was indicated by longer inhibition periods. Even though norV expression is highly upregulated by aerobic NO exposure (Bang et al., 2006), both the norV and nrfA mutants were unaffected by addition of $\mathrm{NO}$ in comparison to the wildtype strain. However, strains with a $h m p A$ mutation showed increased sensitivity to NO (Table 5), and were unable to recover to the same $\mathrm{OD}_{600}$ as the control cultures, agreeing with the well-documented findings that HmpA detoxifies NO aerobically (Crawford \& Goldberg, 1998; Gardner \& Gardner, 2002).

\section{DISCUSSION}

By comparing growth under conditions of NrfA repression and induction, this study has shown that prior synthesis of active NrfA efficiently protects against NO toxicity in anaerobic cultures of $S$. Typhimurium, preventing growth inhibition. Furthermore, $S$. Typhimurium is unable to recover from anaerobic exposure to $\mathrm{NO}$ in the absence of both NrfA and NorV, showing that under these conditions HmpA is unable to protect Salmonella from NO addition. Under aerobic conditions, HmpA is the key enzyme responsible for NO detoxification, in agreement with earlier data (Crawford \& Goldberg, 1998; Gardner \& Gardner, 2002). The ability of NrfA and NorV to contribute to anaerobic NO detoxification has a precedent in E. coli, but has not been observed in a pathogenic 

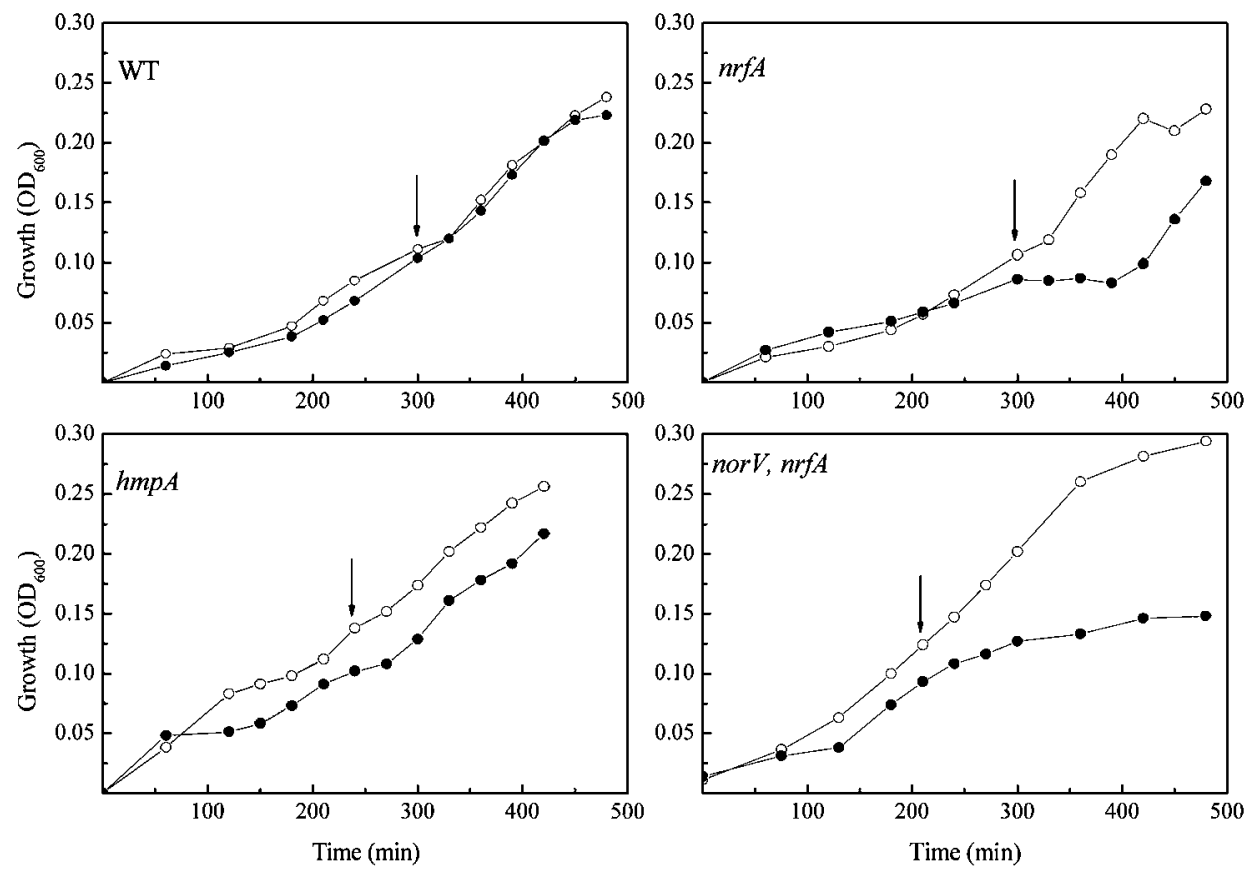

Fig. 4. Representative anaerobic growth curves of wild-type (WT) $S$. Typhimurium and three mutants in GNF medium, a minimal medium with glycerol as the carbon source and nitrate and fumarate as electron acceptors. Duplicate cultures were grown in the absence $(\bigcirc)$ and presence $(0)$ of $40 \mu \mathrm{M}$ NO. NO was added at the time indicated by the arrow and data are representative of three independent experiments. Representative growth curves for all seven mutants are shown in Supplementary Fig. S2.

bacterium. Mutation of the $n r f A$ or norV gene increases the sensitivity of $E$. coli towards $\mathrm{NO}$ under anaerobic conditions (Poock et al., 2002; Gardner et al., 2002; Gomes et al., 2002; Hutchings et al., 2002). The combined action of NrfA and NorV under these conditions provides distinct advantages to the organism. Periplasmic NrfA would metabolize NO prior to its entering the cell. This would reduce the amount of intracellular NO, and any that entered would be reduced by the cytoplasmic NorV.
Furthermore, NrfA is part of the electron-transport chain, and NO detoxification would conserve energy, in contrast to the NADH-dependent activities of NorV and HmpA.

This study has focused on the role of the NorV, NrfA and HmpA enzymes in the survival of $S$. Typhimurium after NO exposure under fermentative and nitrate-respiratory growth conditions, and was designed to assess the differences that arise from the presence of high or low

Table 5. Growth parameters for each mutant grown aerobically in MGM

MGM is a minimal medium with glucose as the carbon source. The doubling times $\left(t_{\mathrm{d}}\right)$ pre and post NO addition are shown, as well as the period of growth inhibition, which was measured as the time between NO addition and a line extrapolated from the growth curve, following recovery, through the intercept of the $\mathrm{OD}_{600}$ at which NO was added. All data are the average of at least three experiments and the SE is shown. Significant growth inhibition is indicated in bold type.

\begin{tabular}{|lccc|}
\hline Strain & $\boldsymbol{t}_{\mathbf{d}}(\mathbf{m i n})$ & $\boldsymbol{t}_{\mathbf{d}}$ post NO (min) & Growth inhibition period (min) \\
\hline S. Typhimurium SL1344 & $46.7 \pm 6.5$ & $51.7 \pm 4.5$ & $29.7 \pm 5.1$ \\
norV & $47.3 \pm 7.8$ & $56.0 \pm 2.0$ & $21.7 \pm 15.0$ \\
nrfA & $47.0 \pm 1.0$ & $54.7 \pm 9.0$ & $26.7 \pm 7.0$ \\
hmpA & $56.7 \pm 3.6$ & $69.0 \pm 3.6$ & $\mathbf{9 4 . 7} \pm \mathbf{2 1 . 5}$ \\
norV, hmpA & $73.7 \pm 20.1$ & $81.3 \pm 19.6$ & $\mathbf{6 2 . 0} \pm \mathbf{9 . 5}$ \\
nrfA, hmpA & $54.7 \pm 4.9$ & $72.1 \pm 7.5$ & $36.3 \pm 8.3$ \\
norV, nrfA & $56.0 \pm 8.4$ & $52.8 \pm 6.1$ & $22.2 \pm 3.8$ \\
norV, nrfA, hmpA & $56.8 \pm 0.8$ & $65.0 \pm 5.0$ & $\mathbf{5 4 . 6} \pm \mathbf{1 . 1}$ \\
\hline
\end{tabular}



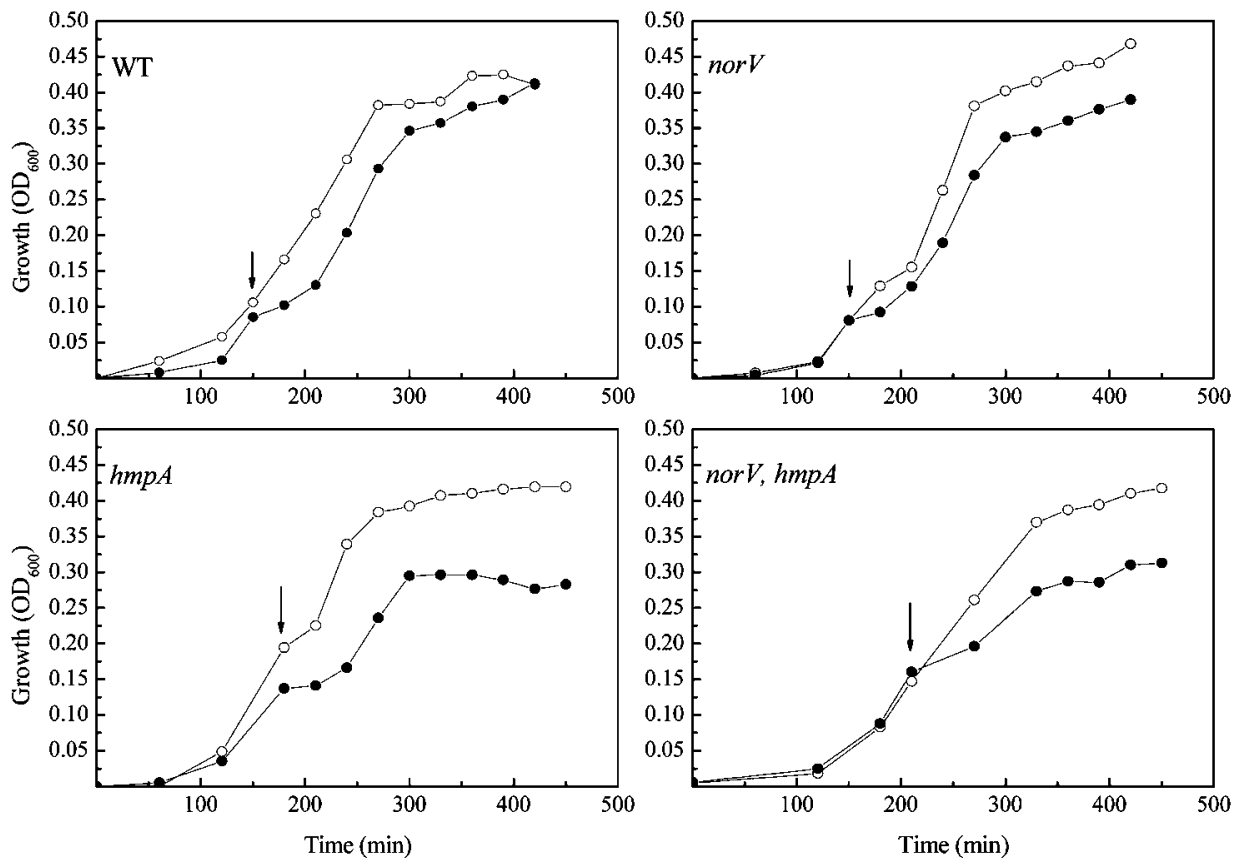

Fig. 5. Representative aerobic growth curves of wild-type (WT) S. Typhimurium and three mutants in MGM, a minimal medium with glucose as the carbon source. Duplicate cultures were grown in the absence $(\bigcirc)$ and presence $(\bullet)$ of $200 \mu \mathrm{M}$ NO. NO was added at the time indicated by the arrow and data are representative of three independent experiments. Representative growth curves for all seven mutants are shown in Supplementary Fig. S3.

endogenous levels of NrfA. It is interesting to consider how this fundamental laboratory study relates to pathogenic bacteria in the outside world. Salmonellae are likely to encounter NO in many different environments, including soil, the gastrointestinal tract, the bloodstream and the macrophage. We speculate that the capacity to express nor $V$, nrfA and $h m p A$ under different conditions provides the metabolic flexibility to protect Salmonella against this cytotoxin in a wide range of oxic and anoxic environments containing different carbon and energy sources and respiratory electron acceptors. Our data suggest that NrfA is not a vital component of the immediate response to NO, but would be important under conditions where NrfA has already been activated. A $n r f A$ mutant is slightly attenuated in a mouse infection model (Bang et al., 2006), suggesting a role for this enzyme in either NO detoxification or anaerobic respiration, both of which have been shown to be important for survival of $S$. enterica serovar Typhi in epithelial cells (Contreras et al., 1997).

HmpA was not required for $S$. Typhimurium to survive exposure to NO under the anoxic conditions used in this in vitro study, and only played a minor role in anaerobic $\mathrm{NO}$ detoxification; this was consistent with some E. coli studies (Gardner \& Gardner, 2002), but not with others (Crawford \& Goldberg, 1998). These discrepancies may reflect the growth conditions used, or whether nitrosated compounds or NO gas were used in the experiments as a source of NO, or both. Earlier research (Gardner \& Gardner, 2002) showed that HmpA was unable to reduce compounds such as GSNO and acidified nitrite directly under in vitro experimental conditions, and so the apparent detoxification effects that were reported earlier may not be due to NO reduction. Our study uses NO, which is the ideal substrate for the study of $\mathrm{NO}$ reduction.

A growing body of evidence suggests that HmpA is important for successful Salmonella infection in mice, and for survival of both Salmonella and E. coli within macrophages (Bang et al., 2006; Gilberthorpe et al., 2007; Stevanin et al., 2002, 2007). Most of this research has focused upon replication inside macrophages grown ex vivo, for which HmpA is undoubtedly vital for the survival of Salmonella. Overall, the fact that multiple enzymes are capable of protecting Salmonella from NO confirms the importance of NO detoxification for Salmonella, and reflects the variety of environmental conditions that are experienced by this bacterium before and during the course of infection. Some of our data strongly indicate that yet more NO-detoxification systems remain to be identified. For example, it is surprising that: (i) the growth of a double hmp nrfA mutant on GNF medium was not inhibited by $\mathrm{NO}$, whereas growth of the single nrfA mutant was inhibited by NO (Table 4); and (ii) recovery from NO addition during aerobic growth of the hmp norV, hmp nrfA and hmp norV nrfA strains was quicker than that of the hmp strain (Table 5). Both observations point towards additional NO-detoxification systems being synthesized in 
the absence of Hmp, and this will be investigated in future studies. Multiple enzymes are also used to survive oxidative stress (Farr \& Kogoma, 1991), and it is likely that the availability of different NO-detoxification strategies for different growth environments is one of the secrets of the success of Salmonella as a pathogen.

\section{ACKNOWLEDGEMENTS}

This work was supported by the UK Biotechnology and Biological Sciences Research Council studentship grant 02B1D08116, and the Core Strategic Grant to J. C. D. H. The authors thank Ann Reilly for her technical support, and Martin Goldberg and Isabelle Hautefort for helpful discussions. D. J.R. thanks the Royal Society and Wolfson Foundation for a Merit Award.

\section{REFERENCES}

Bang, I. S., Liu, L., Vazquez-Torres, A., Crouch, M. L., Stamler, J. \& Fang, F. C. (2006). Maintenance of nitric oxide and redox homeostasis by the Salmonella flavohemoglobin Hmp. J Biol Chem 281, 28039-28047.

Bodenmiller, D. M. \& Spiro, S. (2006). The yjeB (nsrR) gene of Escherichia coli encodes a nitric oxide-sensitive transcriptional regulator. J Bacteriol 188, 874-881.

Contreras, I., Toro, C., Troncoso, G. \& Mora, G. (1997). Salmonella typhi mutants defective in anaerobic respiration are impaired in their ability to replicate within epithelial cells. Microbiology 143, 2665-2672.

Costa, C., Macedo, A., Moura, I., Moura, J., LeGall, J., Berlier, Y., Liu, M.-Y. \& Payne, W. (1990). Regulation of the hexaheme nitrite/nitric oxide reductase of Desulfovibrio desulfuricans, Wolinella succinogenes and Escherichia coli. A mass spectrometric study. FEBS Lett 276, 67-70.

Crawford, M. J. \& Goldberg, D. E. (1998). Role for the Salmonella flavohemoglobin in protection from nitric oxide. J Biol Chem 273, 12543-12547.

da Costa, P. N., Teixeira, M. \& Saraiva, L. M. (2003). Regulation of the flavorubredoxin nitric oxide reductase gene in Escherichia coli: nitrate repression, nitrite induction, and possible post-transcription control. FEMS Microbiol Lett 218, 385-393.

Darwin, A., Hussain, H., Griffiths, L., Grove, J., Sambongi, Y., Busby, S. \& Cole, J. (1993). Regulation and sequence of the structural gene for cytochrome $\mathcal{c}_{552}$ from Escherichia coli: not a hexahaem but a $50 \mathrm{kDa}$ tetrahaem nitrite reductase. Mol Microbiol 9, 1255-1265.

Datsenko, K. A. \& Wanner, B. L. (2000). One-step inactivation of chromosomal genes in Escherichia coli K-12 using PCR products. Proc Natl Acad Sci U S A 97, 6640-6645.

Eriksson, S., Lucchini, S., Thompson, A., Rhen, M. \& Hinton, J. C. (2003). Unravelling the biology of macrophage infection by gene expression profiling of intracellular Salmonella enterica. Mol Microbiol 47, 103-118

Farr, S. B. \& Kogoma, T. (1991). Oxidative stress responses in Escherichia coli and Salmonella typhimurium. Microbiol Rev 55, 561-585.

Field, S. J., Thorndycroft, F. H., Matorin, A. D., Richardson, D. J. \& Watmough, N. J. (2008). The respiratory nitric oxide reductase (NorBC) from Paracoccus denitrificans. Methods Enzymol 437 (in press).

Filenko, N., Spiro, S., Browning, D. F., Squire, D., Overton, T. W., Cole, J. \& Constantinidou, C. (2007). The NsrR regulon of Escherichia coli K-12 includes genes encoding the hybrid cluster protein and the periplasmic, respiratory nitrite reductase. J Bacteriol 189, 4410-4417.

Flatley, J., Barrett, J., Pullan, S., Hughes, M., Green, J. \& Poole, R. (2005). Transcriptional responses of Escherichia coli to S-nitrosoglutathione under defined chemostat conditions reveal major changes in methionine biosynthesis. J Biol Chem 280, 10065-10072.

Gardner, A. M. \& Gardner, P. R. (2002). Flavohemoglobin detoxifies nitric oxide in aerobic, but not anaerobic, Escherichia coli. Evidence for a novel inducible anaerobic nitric oxide-scavenging activity. J Biol Chem 277, 8166-8171.

Gardner, P. R., Gardner, A. M., Martin, L. A. \& Salzman, A. L. (1998). Nitric oxide dioxygenase: an enzymic function for flavohemoglobin. Proc Natl Acad Sci U S A 95, 10378-10383.

Gardner, A. M., Helmick, R. A. \& Gardner, P. R. (2002). Flavorubredoxin, an inducible catalyst for nitric oxide reduction and detoxification in Escherichia coli. J Biol Chem 277, 8172-8177.

Gilberthorpe, N. J., Lee, M. E., Stevanin, T. M., Read, R. C. \& Poole, R. K. (2007). NsrR: a key regulator circumventing Salmonella enterica serovar Typhimurium oxidative and nitrosative stress in vitro and in IFN- $\gamma$-stimulated J774.2 macrophages. Microbiology 153, 1756-1771.

Gomes, C. M., Giuffre, A., Forte, E., Vicente, J. B., Saraiva, L. M., Brunori, M. \& Teixeira, M. (2002). A novel type of nitricoxide reductase. Escherichia coli flavorubredoxin. J Biol Chem 277, 25273-25276.

Hausladen, A., Gow, A. J. \& Stamler, J. S. (1998). Nitrosative stress: metabolic pathway involving the flavohemoglobin. Proc Natl Acad Sci U S A 95, 14100-14105.

Hoiseth, S. K. \& Stocker, B. A. (1981). Aromatic-dependent Salmonella typhimurium are non-virulent and effective as live vaccines. Nature 291, 238-239.

Hutchings, M. I., Mandhana, N. \& Spiro, S. (2002). The NorR protein of Escherichia coli activates expression of the flavorubredoxin gene nor $V$ in response to reactive nitrogen species. J Bacteriol 184, 4640-4643.

Justino, M. C., Vicente, J. B., Teixeira, M. \& Saraiva, L. M. (2005). New genes implicated in the protection of anaerobically grown Escherichia coli against nitric oxide. J Biol Chem 280, 2636-2643.

Kim, S. O., Orii, Y., Lloyd, D., Hughes, M. N. \& Poole, R. K. (1999). Anoxic function for the Escherichia coli flavohaemoglobin (Hmp): reversible binding of nitric oxide and reduction to nitrous oxide. FEBS Lett 445, 389-394.

Membrillo-Hernandez, J., Coopamah, M., Anjum, M., Stevanin, T., Kelly, A., Hughes, M. \& Poole, R. (1999). The flavohemoglobin of Escherichia coli confers resistance to a nitrosating agent, a "nitric oxide releaser", and paraquat and is essential for transcriptional responses to oxidative stress. J Biol Chem 274, 748-754.

Mills, C. E., Sedelnikova, S., Soballe, B., Hughes, M. N. \& Poole, R. K. (2001). Escherichia coli flavohaemoglobin (Hmp) with equistoichiometric FAD and haem contents has a low affinity for dioxygen in the absence or presence of nitric oxide. Biochem J 353, 207-213.

Mukhopadhyay, P., Zheng, M., Bedzyk, L. A., LaRossa, R. A. \& Storz, G. (2004). Prominent roles of the NorR and Fur regulators in the Escherichia coli transcriptional response to reactive nitrogen species. Proc Natl Acad Sci U S A 101, 745-750.

Poock, S. R., Leach, E. R., Moir, J. W., Cole, J. A. \& Richardson, D. J. (2002). Respiratory detoxification of nitric oxide by the cytochrome $c$ nitrite reductase of Escherichia coli. J Biol Chem 277, 23664-23669.

Pope, N. R. \& Cole, J. A. (1984). Pyruvate and ethanol as electron donors for nitrite reduction by Escherichia coli K12. J Gen Microbiol 130, 1279-1284. 
Pullan, S. T., Gidley, M. D., Jones, R. A., Barrett, J., Stevanin, T. M., Read, R. C., Green, J. \& Poole, R. K. (2007). Nitric oxide in chemostat-cultured Escherichia coli is sensed by Fnr and other global regulators: unaltered methionine biosynthesis indicates lack of $\mathrm{S}$ nitrosation. J Bacteriol 189, 1845-1855.

Shiloh, M. U., MacMicking, J. D., Nicholson, S., Brause, J. E., Potter, S., Marino, M., Fang, F., Dinauer, M. \& Nathan, C. (1999). Phenotype of mice and macrophages deficient in both phagocyte oxidase and inducible nitric oxide synthase. Immunity 10, 29-38.

Stach, P., Einsle, O., Schumacher, W., Kurun, E. \& Kroneck, P. M. (2000). Bacterial cytochrome $c$ nitrite reductase: new structural and functional aspects. J Inorg Biochem 79, 381-385.

Stevanin, T. M., Poole, R. K., Demoncheaux, E. A. \& Read, R. C. (2002). Flavohemoglobin Hmp protects Salmonella enterica serovar
Typhimurium from nitric oxide-related killing by human macrophages. Infect Immun 70, 4399-4405.

Stevanin, T. M., Read, R. C. \& Poole, R. K. (2007). The hmp gene encoding the NO-inducible flavohaemoglobin in Escherichia coli confers a protective advantage in resisting killing within macrophages, but not in vitro: links with swarming motility. Gene 398, 62-68.

Vazquez-Torres, A. \& Fang, F. C. (2001). Salmonella evasion of the NADPH phagocyte oxidase. Microbes Infect 3, 1313-1320.

Weinberg, J. (1999). Human mononuclear phagocyte nitric oxide production and inducible nitric oxide synthase expression. In Nitric Oxide and Infection, pp. 95-150. Edited by F. Fang. New York: Kluwer Academic/Plenum Press.

Edited by: S. C. Andrews 